\title{
A Promising Strategy for the Treatment of Onychomycosis with Curcumin and Photodynamic Therapy
}

\author{
Ana Paula da Silva, Fernanda M. Carbinatto, Vanderlei S. Bagnato and Natalia M. Inada \\ University of Sao Paulo, Physics Institute of Sao Carlos, 13566-590 Sao Carlos, SP, Brazil
}

\begin{abstract}
Onychomycosis is a disease of high incidence in the nail plate and responsible for approximately half of the cases of nail infections. Conventionally, dermatologists prescribe antibiotics and antifungals for long periods for its treatment. The high incidence of this type of infection and the increase of microbial strains resistant to the available drugs have justified the importance of the development of new technologies and treatments. This paper presents the photodynamic therapy as an alternative treatment of onychomycosis. New strategies for the use of curcumin as a photosensitizer and its therapeutic response were investigated in different formulations (gel and emulsion). Photodynamic therapy is a promising technique by which microorganisms are eliminated by a photosensitizing compound, light and oxygen. It was evaluated in two patients who had developed lesions in the fingernails caused by onychomycosis for approximately 10 years. The lesions were treated by photodynamic therapy with curcumin. The therapeutic efficacy was observed after a maximum of six photodynamic therapy sessions without any other adjuvant therapy. Curcumin has become more promising than the therapeutic standard as it is a natural and versatible drug for incorporation in different formulations, at low cost and low probability of side effects.
\end{abstract}

Key words: Onychomycosis, photodynamic therapy, curcumin, formulations.

\section{Introduction}

Onychomycosis is a nail fungal infection caused by dermatophytes, yeasts or nondermatophytes. Systemic and topical antifungal agents can be used in its conventional treatment $[1,2]$. However, oral antifungals may cause adverse effects. The topical formulation with antifungal agents may have low therapeutic efficacy because of their limited ability to penetrate the nail plate and reach the affected area [3].These factors and the high incidence of this infection are important parameters for the analyses of

Corresponding author: Natalia M. Inada, Ph.D., doctor of medical pathophysiology, post-doctorate by the physics institute of USP in São Carlos (IFSC), researcher, research fields: biology, medicine, pharmacy, chemistry and physics, with a background in biochemistry (metabolism and bioenergetics), operates mainly in: mitochondrial bioenergetics, tumor cells, reactive oxygen species, cell death mechanisms, basic photodynamic therapy (in vitro and in vivo) and applied (clinical research), optical devices applied in health. E-mail: nataliainada@gmail.com. new formulations associated with other techniques to ensure the success of the therapy.

PDT (photodynamic therapy) is a noninvasive simple technique, therefore it is an interesting therapy for the treatment of onychomycosis. It is characterized by the association of a photosensitizing agent and a light source of suitable wavelength. When this photosensitizer is activated by light in the presence of oxygen, it produces reactive oxygen species that inactivate the fungi and cause onychomycosis [4].

Curcumin is a component obtained from the rizoma of Curcuma Longa Linn and investigated as a potential PS (photosensitizing compound) in PDT for the inactivation of microorganisms. Besides its use as a PS, it can act on localized superficial infections. Initially, curcumin was used mainly in the food industry, but its application is not limited to the food area. It has shown several biological effects, such as suppression of carcinogenesis by avoiding the 
proliferation of a wide variety of tumor cells (skin, lung, stomach, colon and breast), anti-inflammatory, antioxidant and bactericidal activitivies. Pharmacological actions can be intensified when curcumin is irradiated with blue light [5-7].

Silva, A. P. et al reported some promising results regarding the use of curcumin solutions for onychomycosis treatments in patients that had showed unsatisfactory results in conventional therapies and had been affected by the disease for more than 5 years. The patients nail plates were applied a curcumin solution and then illuminated with LED (light emitting diode) $450 \mathrm{~nm}$ [8]. However, curcumin in solution does not remain long in contact with the lesion due to the limitation of its standard formulation. As the nail plate is composed mainly of keratin, a low content of lipids and water, it works as a hydrophilic barrier. Lipids may represent an important route of transportation mainly for hydrophobic substances [9]. As the nail plate hampers the permeation of the PS, we have studied new formulations to improve the success of the therapy with curcumin.

Due to the structural characteristics of the nail plate, curcumin was used in different formulations (hydrophilic gel and water/oil emulsion) for the analysis of the success of the therapy.

\section{Material and Methods}

\subsection{Patient Selection}

The patients with onychomycosis were selected with a clinical diagnosis confirmed by podiatrist.
The experiments were approved by the Ethics Committee of Anhembi Morumbi University (79th Ethics Committee Meeting on November 16, 2010) and patients signed an informed consent.

\subsection{Formulation with PS}

Curcumin was solubilize with $1 \%$ alcohol and $0,1 \%$ dimethyl sulfoxide after Curcumin was incorporated in the $1.5 \%$ concentration in two different formulation, in carbopol gel and emulsion W/O.

\subsection{PDT for Treatment Onycomycosis}

Patients with Onychomycosis were treated for 20 min by an LED (450 nm, $\left.100 \mathrm{~mW} / \mathrm{cm}^{2}\right)$ system developed in the Laboratory of Technological Support and anatomically designed for fingernails and Toenails (total fluence of $120 \mathrm{~J} / \mathrm{cm}^{2}$ ) (Fig. 1).

Patients had their nail plate prepared with a solution of $40 \%$ urea emollient two hours before the treatment. Urea was in contact with the nail for one hour and after this period its excess was removed and PS was applied for one hour before illumination.

Curcumin in the $1.5 \%$ concentration was topically applied in gel or emulsion and subjected to illumination. After a seven-day treatment, a new evaluation was conducted for the analysis of the clinical response to the treatment. The follow-up treatment was performed by a clinical evaluation and documented by photographic images weekly.

For a comparative analysis of different formulations, the treatment was performed in two patients (case 1: male, aged 27 and case 2: female, 65 years old) with

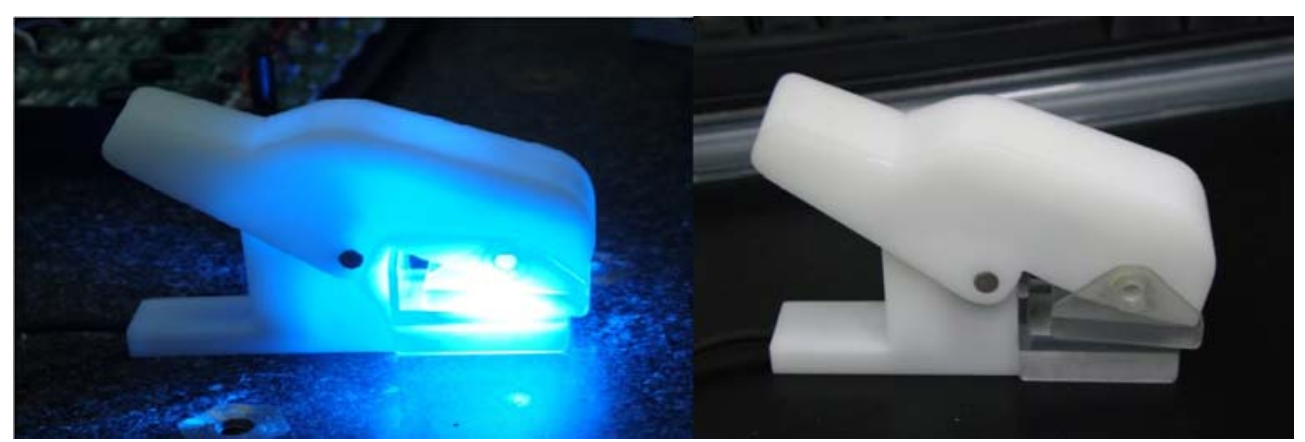

Fig. 1 Design of the device registered in the National Institute of Industrial Property (INPI Protocol No. 18110047225, December 5th, 2011). 
fingernail diseases caused by fungi. Both patients had had the lesions for approximately 10 years. The results were positive for the laboratory cultures, which indicated the presence of fungi.

\section{Results}

3.1 Patient 1: Treatment of Onychomycosis with Gel Curcumin

Gel curcumin (1.5\%) was topically applied to the fingernail (Fig. 2) in case 1. The nail was then protected from light for $1 \mathrm{~h}$. Only 6 PDT sessions were applied and a complete healing was achieved (Fig. 2b) and evidenced by the negative culture of microorganisms for fungi that cause onychomycosis.

\subsection{Patient 2: Treatment of Onychomycosis with} Emulsion Curcumin

Curcumin incorporated in emulsion (1.5\%) was topically applied to the fingernail (Fig. 3) in case 2. The nail was then protected from light for 1 h. Only 5 PDT sessions were necessary and a complete healing was achieved (Fig. 3b) and evidenced by the negative culture of microorganisms for fungi that cause onychomycosis.
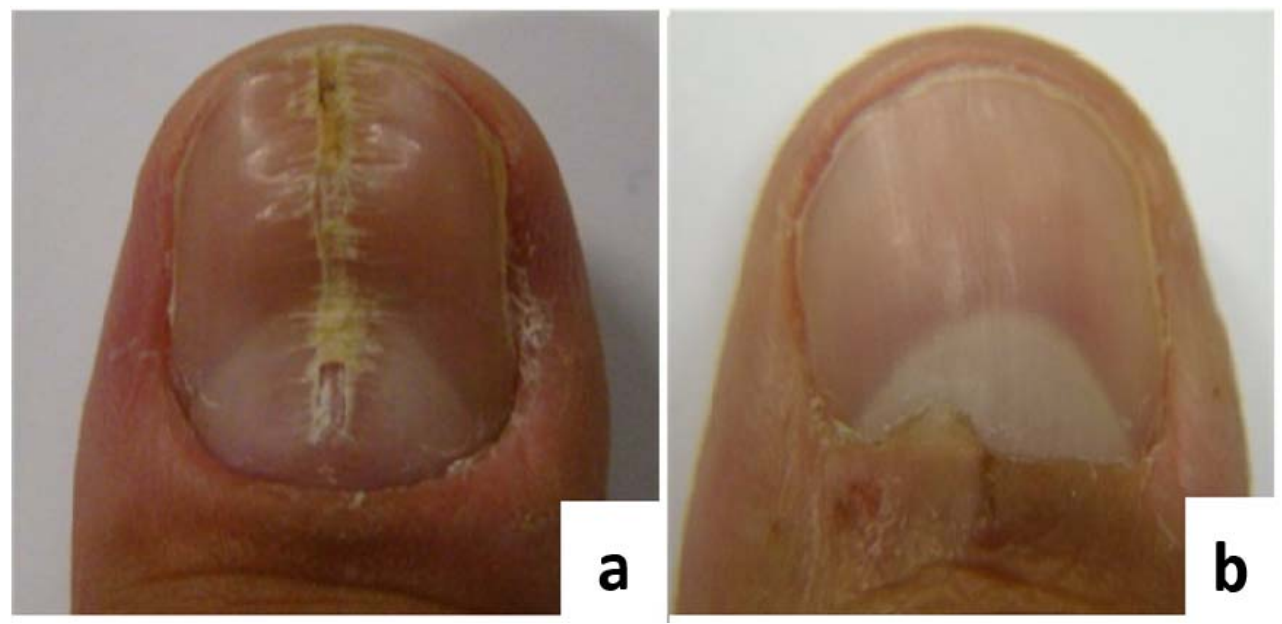

Fig. 2 Male patient, 10 years of lesion, (a) before treatment and (b) after 6 sessions of PDT with curcumin (PDT pharma gel $1.5 \%)$.

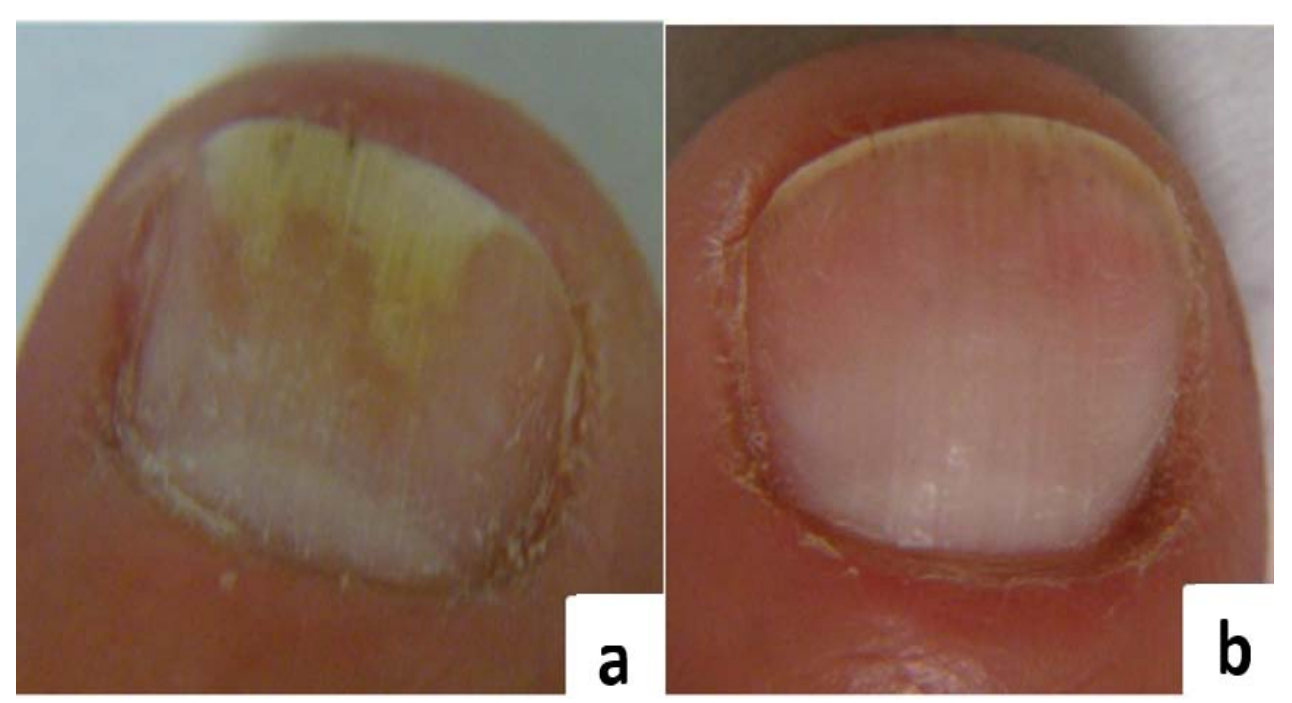

Fig. 3 Female patient, 10 years of lesion, (a) before treatment and (b) after 5 sessions of PDT with curcumin (PDT Pharma Emulsion 1.5\%). 


\section{Discussions}

This study has demonstrated the efficacy of PDT with curcumin as a photosensitizer agent in the treatment of onychomycosis. The possibility of using vehicular curcumin in different formulations (gel and emulsion) can be a positive aspect for this approach.

The new formulation combined with the pre-treatment of the nail bed, can favor the penetration of curcumin in the lesion. The patients reported no pain during and after the treatment and the sessions were repeated weekly.

The satisfactory results achieved with few treatment sessions have proven the differential of this new approach to treat onychomycosis in comparison to conventional treatments, which require a higher number of sessions and may cause discontinuity of the treatment.

\section{Conclusion}

Photodynamic therapy has shown increased potential to aid in microbial and fungal reductions . It was possible to reach a favorable setting for commercial viability of the formulations developed for clinical research, which should help to expedite the availability of this technique to the national and international clinical reality. PDT using different formulation with curcumin is a technique with great potential and low-cost treatment of onychomycosis.

\section{Acknowledgements}

This research has been supported by grants from Fundacão de Amparo à Pesquisa do Estado de São Paulo (FAPESP; CEPOF Grant No. 98/14270-8) and Financiadora de Estudos e Projeto FINEP-Gnatus (Grant No. 554339/2010-2). APS was supported by Conselho Nacional de Desenvolvimento Científico e
Tecnológico (CNPq FINEP-Gnatus; GrantNo. 381132/2010-2).

\section{References}

[1] Naumann, S., Meyer J. P., Kiesow, A., Mrestani, Y., Wohlrab, J., and Neubert, R. H. 2014. "Controlled Nail Delivery of a Novel Lipophilic Antifungal Agent Using Various Modern Drug Carrier Systems as well as in Vitro and ex Vivo Model Systems.” J. Control Rel. 180: 60-70.

[2] Laborde, S. V., and Scher, R. K. 2000. "Developments in the Treatment of Nail Psoriasis, Melanonychia striata, and Onychomycosis.” Dermatol. Clin. 18: 37-46.

[3] Lim, E. H., Kim, H. R., Park, Y. O., Lee, Y., Seo, Y. J., Kim, C. D., Lee, J. H., and Im, M. 2014. "Toenail Onychomycosis Treated with a Fractional Carbon-Dioxide Laser and Topical Antifungal Cream.” J. Amer. Acad. Dermatol. 70: 918-23.

[4] Pavarina, A. C., Ribeiro, A. P. D., Dovigo, L. N. Andrade, C. R. Costa, C. A. S. and Vergani, C. E. 2012. "Photodynamic Therapy to Eradicate Tumor Cells." In: Cell Metabolism-Cell Homeostasis and Stress Response, edited by Dr. Paula Bubulya, InTech, 149-62.

[5] Bruzell, E. M., Morisbak, E., and Tønnesenet, H. H. 2005. "Studies on Curcumin and Curcuminoids. XXIX. Photoinduced Cytotoxicity of Curcumin in Selected Aqueous Preparations.” Photochem. Photobiol. Sci. 4: 523-30.

[6] Wikene, K. O., Hegge, A. B., Bruzell, E., and Tønnesen, H. H. 2014. "Formulation and Characterization of Lyophilized Curcumin Solid Dispersions for Antimicrobial Photodynamic Therapy (aPDT): Studies on Curcumin and Curcuminoids LII.” Drug Dev. Ind. Pharm. 1-9.

[7] Khalil, O. A. K., Oliveira, O. M., Vellosa, J. C., Quadros, A. U., Dalposso, L. M., Karam, T. K., Mainardes, R. M., and Khalil, K. M. 2012. "Curcumin Antifungal and Antioxidant Activities Are Increased in the Presence of Ascorbic Acid.” Food Chem. 133: 1001-5.

[8] Silva, A. P., Chianfrone, D. J., Tinta, J. W. R., Kurachi, C., Inada, N. M., Bagnato, V. S. 2015. "Development and Comparison of Two Devices for Treatment of Onychomycosis by Photodynamic Therapy.” J. Biomed. Opt. 20 (6): 061109.

[9] Murdan, S. 2002. "Drug Delivery to the Nail Following Topical Application.” Int. J. Pharm. 236: 1-26. 